\title{
Note: Accounting for pressure effects on the calculated equilibrium structure of glassy $\mathrm{GeSe}_{2}$
}

Assil Bouzid, and Carlo Massobrio

Citation: The Journal of Chemical Physics 137, 046101 (2012); doi: 10.1063/1.4739953

View online: https://doi.org/10.1063/1.4739953

View Table of Contents: http://aip.scitation.org/toc/jcp/137/4

Published by the American Institute of Physics

\section{Articles you may be interested in}

Origin of structural analogies and differences between the atomic structures of $\mathrm{GeSe}_{4}$ and $\mathrm{GeS}_{4}$ glasses: A first principles study

The Journal of Chemical Physics 143, 034504 (2015); 10.1063/1.4926830

Structure of amorphous GeSeg by neutron diffraction and first-principles molecular dynamics: Impact of trajectory sampling and size effects

The Journal of Chemical Physics 145, 084502 (2016); 10.1063/1.4961265

Oxygen DX center in $\operatorname{In}_{0.17} \mathrm{Al}_{0.83} \mathrm{~N}$ : Nonradiative recombination and persistent photoconductivity Applied Physics Letters 110, 072101 (2017); 10.1063/1.4975934

Investigation of size effects on the structure of liquid $\mathrm{GeSe}_{2}$ calculated via first-principles molecular dynamics The Journal of Chemical Physics 136, 224504 (2012); 10.1063/1.4722101

Band alignment and chemical bonding at the $\mathrm{GaAs} / \mathrm{Al}_{2} \mathrm{O}_{3}$ interface: A hybrid functional study Applied Physics Letters 107, 211601 (2015); 10.1063/1.4936240

Isobaric first-principles molecular dynamics of liquid water with nonlocal van der Waals interactions The Journal of Chemical Physics 142, 034501 (2015); 10.1063/1.4905333

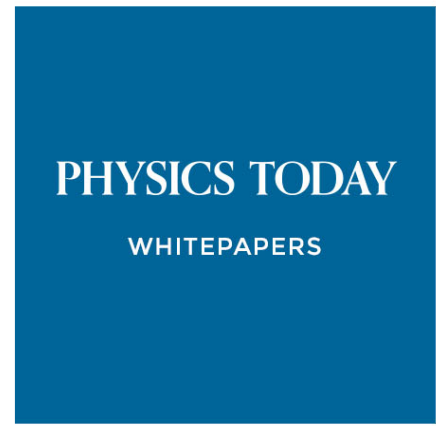

ADVANCED LIGHT CURE ADHESIVES

Take a closer look at what these environmentally friendly adhesive systems can do

\section{READ NOW}

PRESENTED BY Q8 MASTERBOND 


\title{
Note: Accounting for pressure effects on the calculated equilibrium structure of glassy $\mathrm{GeSe}_{2}$
}

\author{
Assil Bouzid and Carlo Massobrio \\ Institut de Physique et de Chimie des Matériaux de Strasbourg, 23 rue du Loess, BP 43, \\ F-67034 Strasbourg Cedex 2, France
}

(Received 15 May 2012; accepted 13 July 2012; published online 31 July 2012)

[http://dx.doi.org/10.1063/1.4739953]

Unambiguous neutron diffraction data have shown that the structure of glassy $\mathrm{GeSe}_{2}(\mathrm{x}=0.33$ composition within the $\mathrm{Ge}_{x} \mathrm{Se}_{1-x}$ family) consists of a variety of structural units, encompassing $\mathrm{GeSe}_{4}$ tetrahedra, homopolar bonds, and defective $\mathrm{Ge}-\mathrm{Se}$ coordinations, accounting for a moderate departure from chemical order. ${ }^{1-3}$ This picture has been substantiated by first-principles molecular dynamics (FPMD) results obtained within the density functional theory (DFT) framework. ${ }^{4,5,7}$ In spite of these successes, it remains to be understood why the number of Ge atoms involved in edgesharing and corner-sharing connections or the number of Se atoms involved in homopolar bonds differ markedly from the experimental results (see Table I). Analysis of the methodology employed within FPMD reveals that most simulations (intended to be stress-free at equilibrium) have been performed at a fixed density. Indeed, no information on the actual values of the pressure is available. ${ }^{5,7}$ Account of the pressure is worthwhile since the density vs pressure relationship holding experimentally might not be exactly reproduced by the DFT model. ${ }^{6}$ As a consequence, undesirable pressure effects altering the room temperature equilibrium properties can be observed. This shortcoming exists regardless of the glass preparation method (quench from the liquid or, much less employed, solid state amorphization).

In this Note, we address this issue via a twofold approach. First, the volume of the periodic system is calibrated at $\mathrm{T}=0 \mathrm{~K}$ by minimizing the non-negligible pressure $(1 / 3$ of the sum of the calculated stress tensor components) acting on the simulated periodic cell of Ref. 7. Then, we performed an extended FPMD simulation at room temperature, thereby allowing the system to adjust its structure to the new pressure conditions. Glassy $\mathrm{GeSe}_{2}$ thereby obtained is more chemically ordered by featuring the correct proportions of $\mathrm{Ge}-\mathrm{Ge}$ homopolar bonds, edge-sharing and corner-sharing connections.

In our theoretical framework, the electronic structure is described within density functional theory and evolves selfconsistently during the motion. ${ }^{8}$ Additional details on our methodology are extensively provided in a previous work. ${ }^{5}$ Calculations on glassy $\mathrm{GeSe}_{2}$ have been carried out in the past at $\mathrm{T}=300 \mathrm{~K}$ for a number density $\rho^{\mathrm{a}}=0.034 \AA^{-3}$ (side length $15.16 \AA$ ), slightly larger than the value quoted in the experimental work $\left(\rho^{\exp }=0.0334 \AA^{-3}\right) .{ }^{9}, 10$ The value of the pressure associated with $\rho^{\mathbf{a}}$ was found to be definitely non-negligible (1 GPa). By expanding the side length to $15.44 \AA$ (density $\rho^{\mathbf{b}}=0.0326 \AA^{-3}$ ) and further relaxing the whole structure, we were able to lower the pressure to less than $\sim 0.1 \mathrm{GPa}$. The final relaxed configuration was selected to begin a thermal annealing cycle, featuring $40 \mathrm{ps}$ at $\mathrm{T}=300 \mathrm{~K}, 50 \mathrm{ps}$ at $\mathrm{T}=600 \mathrm{~K}, 150 \mathrm{ps}$ at $\mathrm{T}=900 \mathrm{~K}$ (to allow significant diffusion) and, on cooling, $70 \mathrm{ps}$ at $\mathrm{T}=600 \mathrm{~K}$ and $150 \mathrm{ps}$ at $\mathrm{T}=300 \mathrm{~K}$, with statistical averages taken over a final portion of the trajectory lasting $20 \mathrm{ps}$ at $\mathrm{T}=300 \mathrm{~K}$. We also performed an additional run (lasting $10 \mathrm{ps}$ ) by employing the constant pressure NPT Andersen technique ${ }^{11}$ with zero pressure as target average value. Standard deviations and instantaneous values for the stress tensor diagonal and non-diagonal terms are not larger than $0.005 \mathrm{GPa}$ and $0.12 \mathrm{GPa}$, respectively, this latter upper bound being much smaller than those calculated for $\rho^{\mathbf{a}}=0.034 \AA^{-3}$. The average density for this NPT calculation is $0.0322 \AA^{-3}$, very close to $\rho^{\mathbf{b}}=0.0326 \AA^{-3}$. Structural properties obtained within the $N V T$ and the NPT trajectory do not differ significantly within statistical errors, estimated from the analysis carried out on Ref. 5 on glassy $\mathrm{GeSe}_{2}$ where as many as six independent trajectories were produced. ${ }^{12}$

In Fig. 1, we display the calculated set of $g_{\alpha \beta}^{\mathrm{a}}(r),{ }^{7} g_{\alpha \beta}^{\mathrm{b}}(r)$ (present results) and experimental $g_{\alpha \beta}^{\exp }(r)$ (Ref. 1) partial pair correlation function, $\alpha, \beta$ being Ge and/or Se. Integrals on the first coordination shell (coordination numbers $n_{\alpha \beta}$ ) are also provided. When comparing $g_{\alpha \beta}^{\mathrm{a}}(r)$ and $g_{\alpha \beta}^{\mathrm{b}}(r)$, small shifts in the peak positions are found within statistical fluctuations (typically of the order of 0.03-0.05 ̊, Ref. 5). On the contrary, the $\rho^{\mathbf{b}}$ data mark a statistically significant improvement for $n_{\alpha \beta}$ (Fig. 1, insets), these properties reflecting both the intensity and the width of the peaks. Focussing on $g_{\mathrm{GeGe}}(r)$, the three main peaks in the region $2 \lesssim r(\AA) \lesssim 4$ have been identified as arising, for increasing $r$, from homopolar $\mathrm{Ge}-$ Ge bonds, Ge atoms involved in edge-sharing connections, and $\mathrm{Ge}$ atoms involved in corner sharing connections, respectively. The new data improve upon those of Ref. 7 and reproduce the experimental trend of the three main peaks intensities in the region $2 \lesssim r(\AA) \lesssim 4$, the second peak being less intense than the first one. As shown in Table I, we obtain an unprecedented agreement for the percentages of $\mathrm{Ge}$ and $\mathrm{Se}$ atoms involved in $\mathrm{Ge}-\mathrm{Ge}$ and $\mathrm{Se}-\mathrm{Se}$ homopolar bonds and for the percentages of $\mathrm{Ge}$ atoms found in edge-sharing and corner-sharing connections. This statement accounts for the statistical uncertainties ( \pm 5 at most in percentage units) that can be attributed to the above quantities (see Ref. 5). Most 


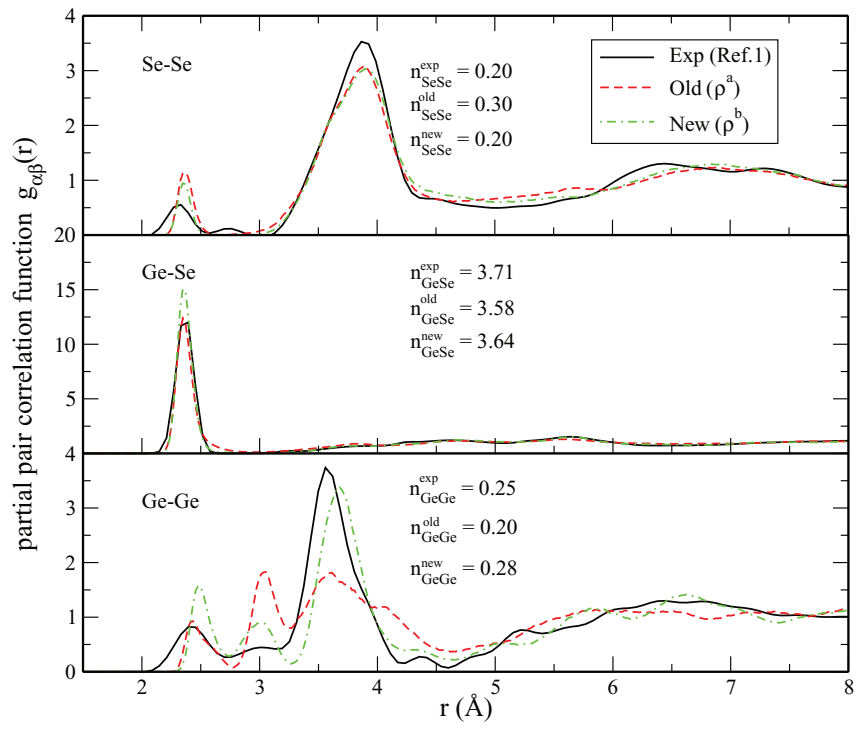

FIG. 1. Partial pair correlation functions for glassy $\mathrm{GeSe}_{2}$. The experimental results of Ref. 1 (solid curve) are compared with the results of Ref. 7 (dashed red curve) and with the present results ( $N V T$ ensemble, dashed-dotted green curve).

remarkable is the improvement in the number of cornersharing connections, stemming from the substantial change in the intensity and shape of the third main peak, in much better agreement with experiments than in the $\rho^{\mathbf{a}}$ case.

Releasing the residual pressure inherent in the previous results for $\rho^{\mathbf{a}}$ has the effect of enhancing the chemical order, with as many as $92 \%$ of Ge atoms being fourfold coordinated, $72 \%$ of them within a $\mathrm{GeSe}_{4}$ tetrahedron. In Ref. 7, these values were equal to $78.1 \%$ and $62.5 \%$, respectively.

Turning to the reciprocal space properties, a correct reproduction of the Bhatia-Thornton ${ }^{13}$ concentrationconcentration partial structure factor $S_{\mathrm{CC}}(k)$ proved to be a stringent test for FPMD approaches. ${ }^{14}$ Observation of Fig. 2 reveals that the intensity of the main peak of $S_{\mathrm{CC}}(k)$, located at $k \sim 2 \AA^{-1}$ is very close to the experimental one for $\rho^{\mathbf{b}}$. This same height was underestimated when working at the $\rho^{\mathbf{a}}$ density (Fig. 2). Also, a minimum is now visible in the range $4 \AA^{-1}<k<5.5 \AA^{-1}$. A detailed analysis (extended to sev-

TABLE I. $N_{\mathrm{Ge}-\mathrm{Ge}}\left(N_{\mathrm{Se}-\mathrm{Se}}\right)$ is the percentage of $\mathrm{Ge}(\mathrm{Se})$ atoms in $\mathrm{Ge}-\mathrm{Ge}$ (Se-Se) homopolar bonds, $N_{\mathrm{Ge}}(\mathrm{ES})$ is the percentage of $\mathrm{Ge}$ atoms forming edge-sharing connections and $N_{\mathrm{Ge}}(\mathrm{CS})$ is the percentage of Ge atoms forming corner-sharing connections. Note that in Ref. 4, a molecular dynamics approach was used in conjunction with a reverse Monte Carlo method.

\begin{tabular}{lcccc}
\hline \hline & $N_{\mathrm{Ge}-\mathrm{Ge}}$ & $N_{\mathrm{Se}-\mathrm{Se}}$ & $N_{\mathrm{Ge}}(\mathrm{ES})$ & $N_{\mathrm{Ge}}(\mathrm{CS})$ \\
\hline Ref. 7 & 20 & 30 & 58 & 22 \\
Ref. 4 & 17 & 30 & 38 & 45 \\
This work & 23 & 18 & 35 & 42 \\
Experiment: Ref. 1 & 25 & 20 & 34 & 41 \\
\hline \hline
\end{tabular}

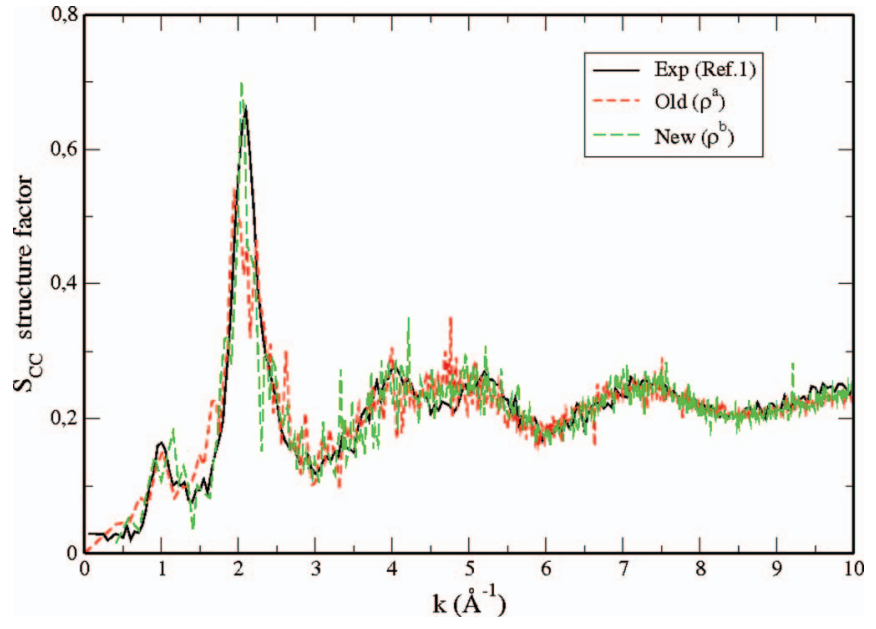

FIG. 2. The Bhatia-Thornton concentration-concentration partial structure factor $S_{\mathrm{CC}}(k)$ for glassy $\mathrm{GeSe}_{2}$. (Solid line) Experimental results of Ref. 1. (Green curve) Present results (NVT ensemble). (Red curve) Results of Ref. 7.

eral independent trajectories) of pressure effects on the partial structure factors of glassy $\mathrm{GeSe}_{2}$ studied within the NVT and NPT ensembles will be made available elsewhere.

In conclusion, account of residual pressure effects on the room temperature, equilibrium structure of glassy $\mathrm{GeSe}_{2}$ leads to a better agreement between atomic-scale models and experiments for this prototypical network-forming disordered material.

This work was performed by using resources from GENCI-(Grand Equipement National de Calcul Intensif) (Grant No. 2012-[095071]).

${ }^{1}$ I. Petri, P. S. Salmon, and H. E. Fischer, Phys. Rev. Lett. 84, 2413 (2000).

${ }^{2}$ P. S. Salmon and I. Petri, J. Phys. Condens. Matter 15, S1509 (2003).

${ }^{3}$ P. S. Salmon, J. Non-Cryst. Solids 353, 2959 (2007).

${ }^{4}$ P. Biswas, D. N. Tafen, and D. A. Drabold, Phys. Rev. B 71, 054204 (2005); D. N. Tafen and D. A. Drabold, ibid. 71, 054206 (2005).

${ }^{5}$ C. Massobrio and A. Pasquarello, Phys. Rev. B 77, 144207 (2008).

${ }^{6}$ D. Alfé, G. D. Price, and M. J. Gillan, Phys. Rev. B 65, 165118 (2002).

${ }^{7}$ C. Massobrio, M. Micoulaut, and P. S. Salmon, Solid State Sci. 12, 199 (2010).

${ }^{8}$ R. Car and M. Parrinello, Phys. Rev. Lett. 55, 2471 (1985).

${ }^{9}$ I. Petri and P. S. Salmon, Phys. Chem. Glasses 43C, 185 (2002).

${ }^{10}$ R. Azoulay, H. Thibierge, and A. Brenac, J. Non-Cryst. Solids 18, 33 (1975).

${ }^{11}$ H. C. Andersen, J. Chem. Phys. 72, 2384 (1980).

${ }^{12}$ In the case of Ref. 5, the exchange-correlation functional due to Perdew and Wang [J. P. Perdew and Y. Wang, Phys. Rev. B 45, 13244 (1992)] was adopted at the place of the Becke, Lee, Yang, and Parr scheme used here [A. D. Becke, Phys. Rev. A 38, 3098 (1988); C. Lee, W. Yang, and R. G. Parr, Phys. Rev. B 37, 785 (1988)]. A comparison between the PW and the BLYP results is given in Ref. 7.

${ }^{13}$ A. B. Bhatia and D. E. Thornton, Phys. Rev. B 2, 3004 (1970).

${ }^{14}$ C. Massobrio and A. Pasquarello, Phys. Rev. B 75, 014206 (2007). 